\title{
Intra-patient stability of tumor mutational burden from tissue biopsies at different time points in advanced cancers
}

Timothy V. Pham ${ }^{1 \dagger}$, Aaron M. Goodman ${ }^{1,2^{*}+}$, Smruthy Sivakumar ${ }^{3}$, Garrett Frampton ${ }^{3}$ and Razelle Kurzrock

\begin{abstract}
Background: Tumor mutational burden (TMB) may be a predictive biomarker of immune checkpoint inhibitor (ICI) responsiveness. Genomic landscape heterogeneity is a well-established cancer feature. Molecular characteristics may differ even within the same tumor specimen and undoubtedly evolve with time. However, the degree to which TMB differs between tumor biopsies within the same patient has not been established.

Methods: We curated data on 202 patients enrolled in the PREDICT study (NCT02478931), seen at the University of California San Diego (UCSD), who had 404 tissue biopsies for TMB (two per patient, mean of 722 days between biopsies) to assess difference in TMB before and after treatment in a pan-cancer cohort. We also performed an orthogonal analysis of 2872 paired pan-solid tumor biopsies in the Foundation Medicine database to examine difference in TMB between first and last biopsies.

Results: The mean (95\% Cl) TMB difference between samples was $0.583[-0.900-2.064](p=0.15)$. Pearson correlation showed a flat line for time elapsed between biopsies versus TMB change indicating no correlation $\left(R^{2}=\right.$ 0.0001; $p=0.8778$ ). However, in 55 patients who received ICls, there was an increase in TMB (before versus after mean mutations/megabase [range] 12.50 [range, 0.00-98.31] versus 14.14 [range, $0.00-100.0$ ], $p=0.025$ ). Analysis of 2872 paired pan-solid tumor biopsies in the Foundation Medicine database also indicated largely stable TMB patterns; TMB increases were only observed in specific tumors (e.g., breast, colorectal, glioma) within certain time intervals.
\end{abstract}

Conclusions: Overall, our results suggest that tissue TMB remains stable with time, though specific therapies such as immunotherapy may correlate with an increase in TMB.

Trial registration: NCT02478931, registered June 23, 2015.

Keywords: Tumor mutational burden, Immunotherapy, Immunotherapy effect on TMB, TMB over time, TMB over treatment

\footnotetext{
* Correspondence: a1goodman@ucsd.edu

†Timothy V. Pham and Aaron M. Goodman contributed equally to this work.

${ }^{1}$ Center for Personalized Cancer Therapy, University of California San Diego

(UCSD), 3855 Health Sciences Drive, La Jolla, CA 92037, USA

${ }^{2}$ Division of Blood and Marrow Transplantation, UCSD, 3855 Health Sciences

Drive, MC-0960, La Jolla, CA 92093, USA

Full list of author information is available at the end of the article
}

(c) The Author(s). 2021 Open Access This article is licensed under a Creative Commons Attribution 4.0 International License, which permits use, sharing, adaptation, distribution and reproduction in any medium or format, as long as you give appropriate credit to the original author(s) and the source, provide a link to the Creative Commons licence, and indicate if changes were made. The images or other third party material in this article are included in the article's Creative Commons licence, unless indicated otherwise in a credit line to the material. If material is not included in the article's Creative Commons licence and your intended use is not permitted by statutory regulation or exceeds the permitted use, you will need to obtain permission directly from the copyright holder. To view a copy of this licence, visit http://creativecommons.org/licenses/by/4.0/ The Creative Commons Public Domain Dedication waiver (http://creativecommons.org/publicdomain/zero/1.0/) applies to the data made available in this article, unless otherwise stated in a credit line to the data. 


\section{Background}

Tumor mutational burden (TMB) is currently emerging as a predictive biomarker of response to checkpoint blockade immunotherapy as noted by the recent Food and Drug Administration approval of the anti-programmed cell death protein (PD-1) antibody pembrolizumab for solid cancers with $\mathrm{TMB} \geq 10$ mutations/Mb [1-4], though there is still controversy regarding its value due to its variable distribution depending on cancer type [5-8], sequencing methodology, and lack of standards for the definition of "high," "medium," or "low" TMB, and the consequent lack of harmonized clinical validation [9]. Some studies have shown TMB to be a more robust biomarker for its correlation with outcome than simply programmed death ligand 1 (PD-L1) expression alone [10], which is one of the current National Comprehensive Cancer Network $(\mathrm{NCCN})$ criteria for prescribing checkpoint blockade immunotherapy [11].

Checkpoint blockade immunotherapies target a tumor immune evasion mechanism by interfering with the interaction between tumor-expressed PD-L1 and PD-1 expressed on cytotoxic T-cells. Logically, to be a valid attack vector, a $\mathrm{T}$ cell must first be able to recognize the cancer cell as foreign through the peptide antigens the tumor cell presents on its surface, themselves a product of the tumor's genome, which may be mutated due to factors such as exposure to environmental carcinogens like tobacco smoke and ultraviolet radiation, as well as dysregulated endogenous mechanisms such as apolipoprotein B mRNA-editing enzyme and catalytic polypeptide-like (APOBEC) enzyme-mediated kataegis [12-15]. The more mutations (and hence the higher the TMB), presumably the better the chance for immune recognition [13].

There has been scant analysis regarding if and how a tumor's TMB changes over the course of disease and treatment. However, Gerlinger et. al. [16] showed that tumors are tremendously heterogenous even within the same patient [16]. Further, Riaz et al. [17] reported that TMB decreases in melanoma when immunotherapy treatment is successful and that certain subclonal populations within the tumor are eliminated due to the action of tumor infiltrating lymphocytes (TIL) activated by the checkpoint inhibitor nivolumab, changing the tumor's composition and therefore post-treatment immune microenvironment [17].

Our aim was to determine if TMB changed over time in patients with repeated biopsies. We found that TMB across cancer types remained stable between biopsies and any differences in TMB did not correlate with time elapsed between biopsies. However, within specific tumor types and/or with specific therapies such as immunotherapy, an increase in TMB with time was observed.

\section{Methods}

\section{Patient and sample demographics}

We examined clinical data, specifically diagnosis and oncology drug treatments with associated dates and outcomes, sex, and dates of birth and last follow-up or death, from 225 University of California San Diego (UCSD) patients enrolled in the UCSD PREDICT study (Trial Registration NCT02478931, https://clinicaltrials. gov/ct2/show/NCT02478931) who had $\geq 2$ biopsies that underwent next-generation sequencing (NGS). This was a non-therapeutic, correlative study of personalized medicine with retrospective and prospective components. Patient medical records are examined for results of molecular profiling obtained through standard of care testing to help understand, in a descriptive fashion, how well molecular testing might predict response to therapy. Patient outcome parameters including, but not limited to, tumor response, time to treatment failure, patient survival, and toxicity were analyzed, as well as pharmacodynamic (PD) and pharmacokinetic (PK) data when available. This study also included research-related testing of tissue, blood, or urine specimens via a variety of simple or advanced techniques such as molecular, proteomic, and metabolic analyses for biomarker discovery or for PK and PD parameters.

Eligibility and inclusion/exclusion critereria were: All patients with a diagnosis of cancer or cancer-related referred to a UCSD Health System facility, Eisenhower Medical Center (EMC), and Rady Children's Hospital San Diego (RCHSD) were eligible. Patients or their legal guardians must be willing and able to provide written informed consent to participate in the prospective part of the study unless the patient has been lost to clinical follow-up.

The data was curated from patient charts stored on the UCSD Moores Cancer Center Electronic Medical Records system. Data on the patients' somatic mutations, $\mathrm{TMB}$, and microsatellite status were obtained from the NGS reports provided by Foundation Medicine. From there, only patients whose NGS reports were from a tumor of the same histology with at least two valid, numerical TMBs, who had valid diagnosis data and distinct test dates were selected, leaving 202 patients in total (Additional file 1: Fig. S1, CONSORT Diagram). The difference in TMB between the earliest and latest biopsies, in chronological order, was quantified for further analysis. The 202 UCSD patients were then dichotomized into groups representing drug type exposure (immunotherapy and non-immunotherapy), and the TMB difference between the groups was tested for statistical significance. This study was performed in accordance with UCSD IRB guidelines for the PREDICT protocol (NCT02478931, https://clinicaltrials.gov/ct2/show/ NCT02478931) and for any investigational treatments or 
procedures for which patients gave consent. Research followed the Declaration of Helsinki guidelines. Waiver of consent was permitted by protocol and UCSD IRB for non-identifying retrospective data collection and data analysis. The de-identified UCSD patient data with all variables used in the analysis is available in Additional file 2: Table S2. The study protocol for the PREDICT study is available in Additional file 3: PREDICT Study Protocol. The consent form for the PREDICT study is available in Additional file 3: PREDICT Consent Form.

In addition to the dataset above, we assessed paired tumor biopsy samples from 2872 patients (Additional file 2: Table S1, Additional file 2: Table S3) who underwent targeted comprehensive genomic profiling as part of routine clinical care at Foundation Medicine, as described previously in Frampton et al [18]. For patients with more than two available samples, we included only the first and last sample for paired TMB assessments. We further examined tumor types with at least 50 pairs and binned the collection time difference between the biopsies into the following groups: $\leq 365$ days, 366 to 1095 days, and $>1095$ days. Approval for the Foundation Medicine study including a waiver of informed consent and a Health Insurance Portability and Accountability Act (HIPAA) waiver of authorization was obtained from the Western Institutional Review Board (Protocol No. 20152817). The de-identified Foundation Medicine patient data with all variables used in the previously described analysis is available in Additional file 2: Table S3.

\section{TMB evaluation}

In both the UCSD and Foundation Medicine cohorts, mutations in each sample were identified using hybridcapture-based NGS on formalin-fixed paraffin embedded (FFPE) tumor samples by Foundation Medicine's clinical laboratory improvement amendments (CLIA)-certified lab, specifically with the FoundationOne, FoundationOne ${ }^{\circ} \mathrm{CDx}$, or FoundationOne Heme panels containing $182,236,315$, or 406 genes depending on the date of sequencing and test type, as previously described in [18] (Foundation Medicine, Cambridge, MA, USA; http:// www.foundationone.com/). These different panel types were used in order to maximize the amount of available data due to the multi-year and multi-diagnosis nature of the study. On average, the sequencing depth for $>99 \%$ of exons was greater than $100 x$, for a total depth of coverage of at least 250x covering 1.2 Megabases (Mb). The numerical TMB in mutations/Mb was assessed by extrapolating the number of mutations captured by the panel to the whole genome with a validated algorithm [19]. Germline and characterized oncogenic alterations were not included. The biopsied samples were trichotomized into three groups of low ( $\leq 5$ mutations/Mb), intermediate (between 6 and 19 mutations/Mb, inclusive), and high ( $\geq 20$ mutations/Mb) TMB [20].

\section{Statistical analysis}

A Wilcoxon signed-rank test (non-parametric paired test) was used to compare the earlier TMB with the later TMB due to the linked nature of the before-and-after comparison. Statistical significance for the effects of immunotherapy on TMB change was determined using a 2-tailed Mann-Whitney $U$ test. Correlation between time elapsed between biopsies and measured TMB difference was assessed with linear regression and the $R^{2}$ of the Pearson correlation was reported to show strength of correlation. $p$ values of $\leq 0.05$ were considered statistically significant. These statistical tests were performed using GraphPad Prism version 6.01 (San Diego, CA, USA).

\section{Results}

In the UCSD clinically curated cohort of 202 patients with 404 tissue biopsies for TMB (two biopsies per patient), the average TMB change over the course of treatment, regardless of agent, was 0.583 mutations/Mb (95\% CI - 0.900-2.064 mutations/mb), with an average time between biopsies of 722 days (95\% CI 621-821 days) (Table 1). There was no significant difference in TMB between the earlier and later biopsies $(p=0.15)$ (Fig. 1c). The slope of the linear regression between earlier and later biopsies is significant $(p<0.0001)$ with a moderate Pearson correlation of $R^{2}=0.459$ (Fig. 1a). Additional file 1: Fig. S2a and S2b also show that this correlation is stable if we analyze TMB 0 to 20 mutations/mb or if we $\log$ transform the TMB to attenuate the influence of outliers.

There was also no correlation (Pearson $R^{2}=0.0001$ ) between time elapsed between biopsy and TMB difference (Fig. 1b), and the slope of the line of best fit for time elapsed between biopsy and TMB difference is also not significantly different than zero ( $p=0.8778$ ) (Fig. 1b).

In the 55 patients who received checkpoint blockade immunotherapy between biopsies, the mean (range) $\mathrm{TMB}$ in mutations/mb before immunotherapy was 12.50 (range 0.00 to 98.31 ) versus 14.14 (range 0.00 to 100.0) after immunotherapy ( $p=0.025$ ) (Table 2). Patients exposed to immunotherapy between biopsies $(N=55)$ had an average TMB change of 1.641 mutations/Mb (95\% CI - 3.492-6.775) mutations/mb whereas those without had an average TMB change of - 0.166 (95\% CI 1.524-1.193) mutations $/ \mathrm{mb}$, a significant difference $(p=$ 0.037) (Fig. 1d, Table 2). If the 14 patients who had breast, colon, and ovarian cancer or gliomas (the diseases that showed some increase in TMB with time in the larger Foundation Medicine dataset (Fig. 2)) were eliminated from this analysis, there was still a significant 
Table 1 Demographic/biopsy data of UCSD patient cohort with evaluable TMBs for multiple biopsies and clinical data

\begin{tabular}{|c|c|c|c|c|}
\hline Factor & \multicolumn{3}{|l|}{ Item } & Value $[95 \% \mathrm{Cl}]$ \\
\hline & \multicolumn{3}{|c|}{ Average time between biopsies (days) } & $722[621-821]$ \\
\hline & \multicolumn{3}{|c|}{ Average TMB difference (mutations/Mb) } & $0.583[-0.900-2.064]$ \\
\hline & \multicolumn{3}{|c|}{ Age at first biopsy (years) } & $57.36[55.22-59.50]$ \\
\hline \multirow[t]{2}{*}{ Gender } & \multicolumn{3}{|l|}{ Men } & $106(52.48 \%)$ \\
\hline & \multicolumn{3}{|l|}{ Women } & $96(47.52 \%)$ \\
\hline \multirow[t]{4}{*}{ Diagnoses } & \multicolumn{3}{|c|}{ Colorectal adenocarcinoma } & $29(14.36 \%)$ \\
\hline & \multicolumn{3}{|c|}{ Lung adenocarcinoma } & $20(9.901 \%)$ \\
\hline & \multicolumn{3}{|c|}{ Breast invasive ductal adenocarcinoma } & $10(4.950 \%)$ \\
\hline & \multicolumn{3}{|c|}{ Metastatic (any primary) } & $99(49.00 \%)$ \\
\hline \multirow[t]{7}{*}{ Biopsy sites } & \multicolumn{2}{|l|}{ First biopsy } & \multicolumn{2}{|l|}{ Last biopsy } \\
\hline & Lung & $22(10.89 \%)$ & Liver & $33(16.34 \%)$ \\
\hline & Colon & $19(9.406 \%)$ & Lung & $20(9.901 \%)$ \\
\hline & Soft Tissue & $16(7.921 \%)$ & Lymph Node & $16(7.921 \%)$ \\
\hline & Bone Marrow & $11(5.446 \%)$ & Bone Marrow & $14(6.931 \%)$ \\
\hline & Liver & $11(5.446 \%)$ & Colon & $12(5.941 \%)$ \\
\hline & Others & $123(60.89 \%)$ & Others & $107(52.97 \%)$ \\
\hline \multirow[t]{2}{*}{ Immunotherapy (checkpoint) } & \multicolumn{3}{|c|}{ Number receiving immunotherapy between biopsies } & $55(27.23 \%)$ \\
\hline & \multicolumn{3}{|c|}{ Number NOT receiving immunotherapy } & $147(72.77 \%)$ \\
\hline \multirow[t]{6}{*}{ TMB measured } & \multicolumn{3}{|c|}{ High at first biopsy ( $\geq 20$ mutations/Mb) } & $10(4.950 \%)$ \\
\hline & \multicolumn{3}{|c|}{ Intermediate at first biopsy ( $5<\mathrm{TMB} \leq 19$ mutations/Mb) } & $49(24.26 \%)$ \\
\hline & \multicolumn{3}{|c|}{ Low at first biopsy ( $\leq 5$ mutations/Mb) } & $143(70.79 \%)$ \\
\hline & \multicolumn{3}{|c|}{ High at second biopsy ( $\geq 20$ mutations/Mb) } & $13(6.436 \%)$ \\
\hline & \multicolumn{3}{|c|}{ Intermediate at second biopsy ( $5<\mathrm{TMB} \leq 19$ mutations/Mb) } & $50(24.75 \%)$ \\
\hline & \multicolumn{3}{|c|}{ Low at second biopsy ( $\leq 5$ mutations/Mb) } & $139(68.81 \%)$ \\
\hline
\end{tabular}

Patients in the UCSD cohort $(N=202)$ tend to be older and are roughly evenly distributed between men and women. Almost half $(49 \%)$ of the patients have metastatic cancer. 55 of them (27.23\%) received immunotherapy between their first and last biopsies, on average 722 days apart

Data derived from medical record; if tobacco or alcohol exposure not known, then patients not counted

increase in TMB with time in the immunotherapytreated 41 patients without these diagnoses $(p=0.0202)$

Of the 2872 pairs of TMB samples from the same patient in the Foundation Medicine database, we examined disease groups with at least 50 pairs: breast cancer $(N=$ 633 pairs), colorectal $(N=423)$, non-small cell lung cancer $(N=423)$, ovarian cancer $(N=249)$, glioma $(N=218)$, esophageal cancer $(N=116)$, melanoma $(N=89)$, prostate cancer $(N=87)$, and endometrial cancer $(N=87)$ (Fig. 2 , Additional file 2: Table S1, Additional file 1: Fig. S3, Additional file 1: Fig. S4). In the 2872 paired samples, the median collection time between samples was 448 days (range, 0 to 5444 days). The average change of TMB over time was 1.58 mutations/mb (95\% confidence interval [CI], 1.04 to 2.12 mutations/mb). Overall, there was no correlation between time elapsed and TMB change (Pearson correlation coefficient $=0.041, p=0.03$ ) (Additional file 1 : Fig. S3A, B). However, certain tumor types showed significant difference in TMB change between the different binned collection time differences (Fig. 2). Breast and colorectal tumors showed statistically significant differences between the collection time bins ( $\leq 365$ days) and (>1095 days) as well as between the collection time bins (366-1095 days) and (> 1095 days), whereas gliomas showed a statistically significant difference relatively early, between collection time bins ( $\leq 365$ days) and (366-1095 days). TMB values at earlier and later biopsies were also well-correlated with one another, with linear regression slopes approaching 1 and significantly non-zero (all $p<1$ $\times 10^{-4}$ ) when each histology group was analyzed separately (Additional file 1: Fig. S3C), and for all paired biopsy samples in the Foundation Medicine, samples were analyzed together (Additional file 1: Fig. S3D). When the TMB values were logarithmically transformed, the results remained consistent, meaning that outlier TMB values did not substantially influence the results (Additional file 1: Fig. S3E, F). 


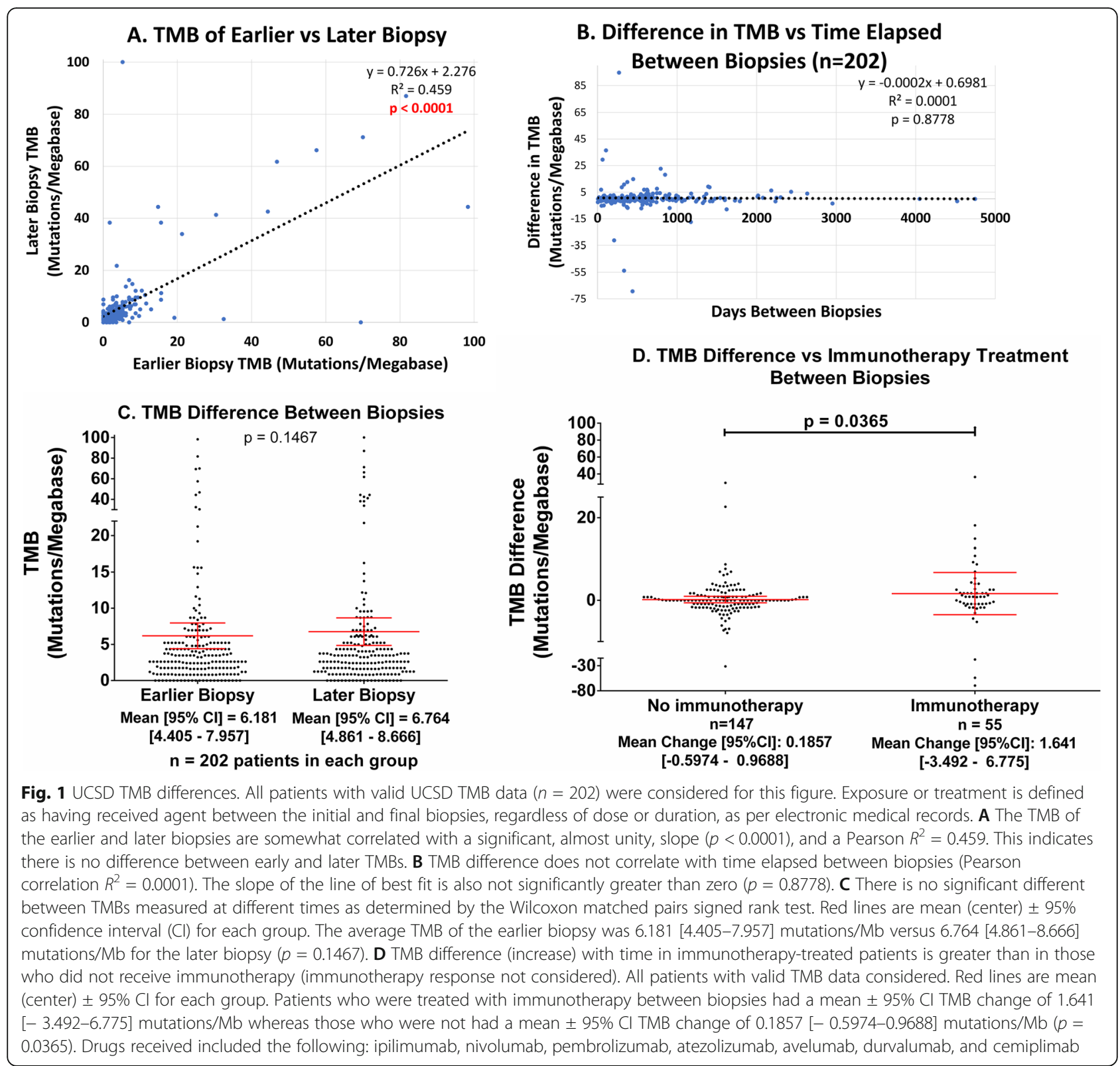

Table 2 Immunotherapy exposure between biopsies and effects on TMB in UCSD cohort $(N=202)$

\begin{tabular}{|c|c|c|c|c|}
\hline \multirow[t]{2}{*}{ Drug } & \multicolumn{3}{|l|}{ Mean TMB [95\% CI] (mutations/Mb) } & \multirow{2}{*}{$\begin{array}{l}\text { Number } \\
\text { receiving/total }\end{array}$} \\
\hline & Before & After & $\overline{p \text { value }}$ & \\
\hline \multirow[t]{3}{*}{ Immunotherapy } & $12.50[6.565-18.43]$ & $14.14[7.960-20.32]$ & 0.0252 & $55 / 202(27.23 \%)$ \\
\hline & $\begin{array}{l}\text { Mean }[95 \% \mathrm{CI}] \text { TMB difference (mutations/Mb), } \\
\text { with immunotherapy } \\
(n=55)\end{array}$ & $\begin{array}{l}\text { Mean }[95 \% \mathrm{Cl}] \mathrm{TMB} \text { difference } \\
\text { (mutations/Mb), no immunotherapy } \\
(n=147)\end{array}$ & $p$ value & \\
\hline & $1.641[-3.492-6.775]$ & $0.186[-0.597-0.969]$ & 0.0365 & \\
\hline
\end{tabular}

Difference in TMB between first and last biopsies were compared for patients exposed to medication, in any amount, between biopsy dates versus difference in TMB between first and last biopsies for all patients who did not receive the specified medication(s) between the biopsy dates. Only the 202 patients with valid TMB data were considered. The results show a significant difference $(p<0.05)$ in TMB difference and overall TMB before and after immunotherapy use 


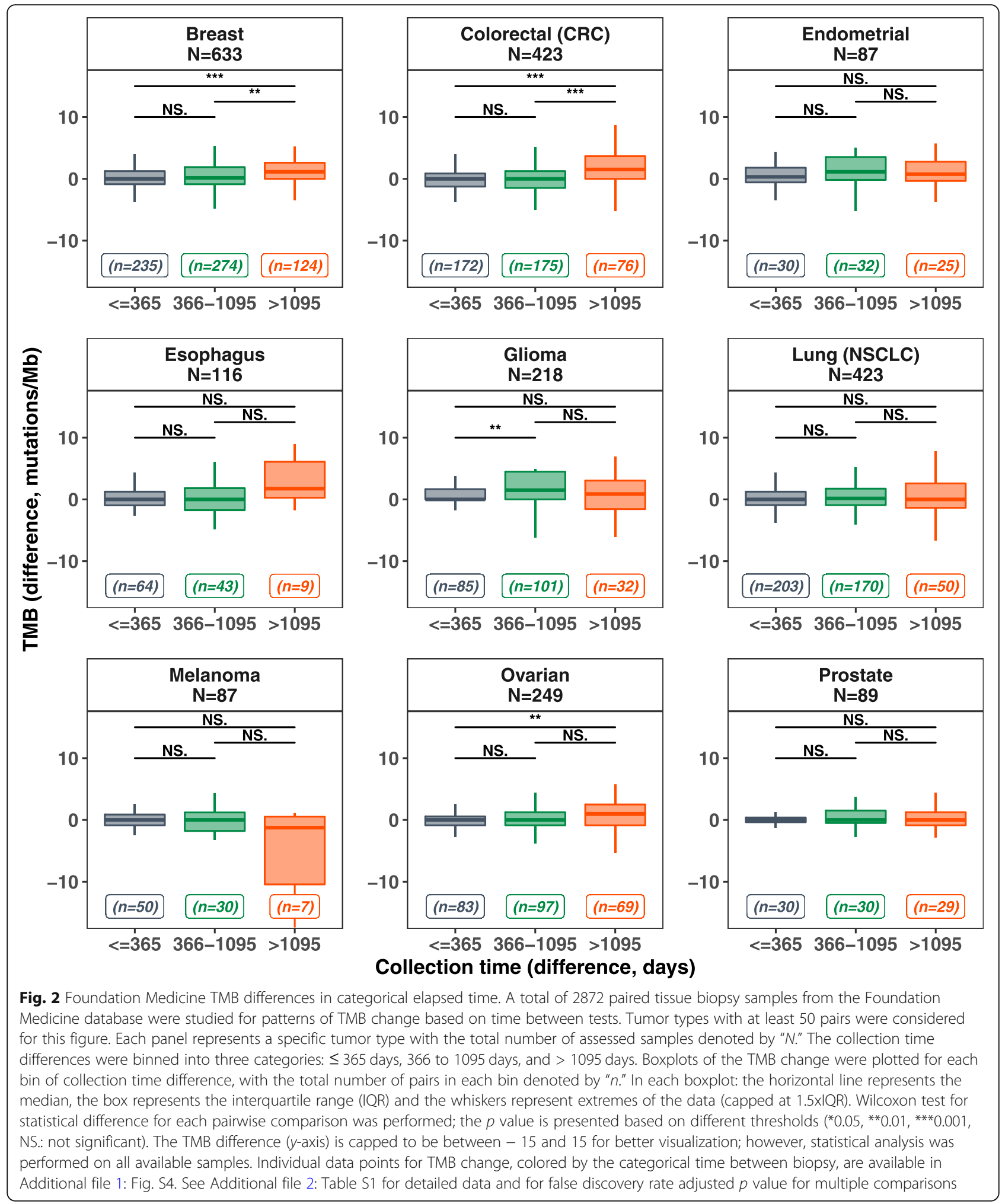

\section{Discussion}

TMB is a quantification of molecular alterations, often expressed in mutations per megabase and determined via genomic sequencing methods such as whole genome or exome sequencing [3] or extrapolation from sequencing part of the genome. TMB may reflect to what extent a tumor's cells will present mutated antigens that the host's immune system can recognize and bind to, in 
order to be primed for checkpoint blockade immunotherapy. Much has been written about TMB's potential as a positive predictor of response pre-treatment $[3,21$, 22] in cohorts of specific cancers such as lung [22], melanoma, and glioblastoma [23], as well as in pan-cancer cohorts $[1,24]$, though not all studies agree and the predictive power of TMB for immunotherapy response remains a matter of debate. Still, the reason behind checkpoint blockade's resounding success in melanoma may be because melanoma is characterized by a high mutational burden [14]. High TMB is correlated with disabling mutations in mismatch repair genes such as MSH2, MSH6, MLH1, POLE, PMS2, and POLD1 [23], which is logical since mutations are a product of DNA repair deficiencies; further, mismatch repair defects are themselves another potential marker of immunotherapy response [25]. Higher TMB is associated with higher neo-antigen load, perhaps explaining the immune responsiveness.

Molecular heterogeneity, even in the same tumor sample as well as those emanating from the same primary, but separated spatially and temporally, has been well described and confounds genomic-based treatments [16]. However, to date, to our knowledge, whether or not heterogeneity in TMB exists as well has not been investigated. Our study shows that, in 2872 pairs of TMB samples from the same patient in the Foundation Medicine database, with a median collection time between samples of 448 days, there was no correlation between time elapsed and TMB change. Importantly, however, TMB changes were observed in specific tumor types within the Foundation Medicine cohort: breast and colorectal tumors and gliomas showed statistically significant TMB increases between certain time bins, with gliomas showing these significant increases in TMB relatively early. Similarly, in 202 clinically curated UCSD patients from the PREDICT study with two tissue biopsies separated by a mean of 722 days, there was no material change in TMB across tumor types. Further, the time interval between biopsies had virtually no effect on the TMB level. However, patients that had received checkpoint blockade between biopsies did show a statistically significant increase in TMB. The latter results differ from those previously published by Riaz et al. [17] who reported that TMB decreases in melanoma when immunotherapy treatment is effective. Of note, we did not sort our patients by response, in part because only 55 immunotherapy-treated patients were available. The fact that non-responders were included in our cohort (and the majority of patients do not respond to immunotherapy) may easily explain the differing results between our study and that of Riaz et al. [17]. Even so, these observations require validation in by other investigators, as the numerical increase between time points is small. Of note, the TMB changes observed in specific tumor types within the large Foundation Medicine cohort may potentially, in part, be attributed to therapy as well. For example, the observed TMB increase in gliomas could be reflective of treatment with temozolomide, a previously known mechanism [26]. However, the lack of treatment information for this cohort makes it unfeasible to test for such patterns. Finally, there were individual patients with large positive or negative swings in TMB; these individuals were disproportionately represented in the immunotherapy-treated cohort.

Our observations have several limitations, including their retrospective nature and the fact that, in the UCSD clinically curated database, because of the heterogeneity of treatments in this group of individuals, the confounding influence of therapies other than immunotherapy was not addressed, and requires a prospective study. Single cell resolution sequencing data, which would have been necessary to distinguish subclonal characteristics within each patient such as TMB, mutation, and expression patterns, and could have provided biological explanation for our observations, was not available to us. Moreover, binning of time periods was based on availability of samples, and even larger sample sizes with different bins might produce additional information. Finally, the large Foundation Medicine dataset did not have clinical therapy curated (though this was curated for the UCSD dataset) and could be biased by selection based on which patients had sequencing performed in the first place, and which patients survived and had a second test done. The lack of information about therapies used, biopsy sites, and lack of subclonal information also limits the translational utility of the data set. Despite these limitations, both the UCSD and Foundation Medicine cohorts show that TMB is largely invariant over time.

\section{Conclusions}

In summary, we find that TMB remains stable between tumor biopsies regardless of time interval between sampling. However, in specific tumor types such as breast, colorectal cancer, and gliomas, there may be increases in TMB with time. Furthermore, individual therapies such as immunotherapy may be associated with an overall increase in TMB.

\section{Abbreviations}

TMB: Tumor mutational burden; N: Number; Cl: Confidence interval; PD1: Programmed cell death protein 1; PD-L1: Programmed death ligand 1; NCCN: National Comprehensive Cancer Network; APOBEC: Apolipoprotein B mRNA-editing enzyme, catalytic polypeptide-like; TIL: Tumor infiltrating lymphocyte; NGS: Next-generation sequencing; FFPE: Formalin-fixed paraffin embedded; CLIA: Clinical laboratory improvement amendments; $\mathrm{Mb}$ : Megabase 


\section{Supplementary Information}

The online version contains supplementary material available at https://doi. org/10.1186/s13073-021-00979-8.

Additional file 1: Figure S1. CONSORT Diagram for UCSD clinically curated cohort. Figure S2. Additional correlation analysis between earlier and later TMB for UCSD clinically curated cohort. Figure S3. Linear Regression Analysis of Paired Biopsies in the Foundation Medicine Cohort. Figure S4. Difference in TMB from paired biopsies in Foundation Medicine Cohort

Additional file 2: Foundation Medicine and UCSD Cohort Characteristics. Table S1. Patterns of TMB difference based on time between biopsies in the Foundation Medicine cohort. Table S2. Patients with paired tissue biopsy samples in the UCSD cohort. Table S3. Patients with paired tissue biopsy samples in the Foundation Medicine cohort

Additional file 3:. PREDICT Study Protocol and Consent Form. Study Protocol. Consent Form

\section{Acknowledgements}

Not applicable.

\section{Authors' contributions}

TP and AG performed the statistical analysis on the UCSD PREDICT patient cohort. SS and GF performed the statistical analysis on the Foundation Medicine cohort. RK conceptualized and supervised this study. All authors contributed to writing the text of the manuscript. All authors read and approved the final manuscript.

\section{Funding}

This work was funded in part by the Joan and Irwin Jacobs Fund and NIH P30 CA023100 (RK) as well as NIH grants U01CA217885, U24CA220341, P30CA023100, and U24CA248457 (TP).

\section{Availability of data and materials}

De-identified data of the variables used for statistical analysis of the 202 patients from the UCSD PREDICT cohort are available in Additional file 2 : Table S2. De-identified, consented data for the 2872 patients in the Foundation Medicine cohort analyzed in the current study are included in Additional file 2: Table S3. All patients/participants in this study did not consent to release of raw sequence data for confidentiality or privacy purposes/in compliance with the ethics approvals obtained for this study.

\section{Declarations}

\section{Ethics approval and consent to participate}

This study was performed in accordance with UCSD IRB guidelines for the PREDICT protocol (NCT02478931) and for any investigational treatments or procedures for which patients gave consent. Waiver of consent was permitted by protocol and UCSD IRB for non-identifying retrospective data collection and data analysis.

Approval for the Foundation Medicine study including a waiver of informed consent and a Health Insurance Portability and Accountability Act (HIPAA) waiver of authorization was obtained from the Western Institutional Review Board (Protocol No. 20152817)

Written informed consent was obtained from all participants.

The research followed the Declaration of Helsinki guidelines.

\section{Consent for publication}

Not applicable.

\section{Competing interests}

RK has the following disclosure information: Stock and Other Equity Interests (IDbyDNA, CureMatch, Inc., and Soluventis); consulting or advisory role (Gaido, LOXO, X-Biotech, Actuate Therapeutics, Roche, NeoMed, Soluventis, and Pfizer); speaker's fee (Roche); research funding (Incyte, Genentech, Merck Serono, Pfizer, Sequenom, Foundation Medicine, Guardant Health, Grifols, Konica Minolta, DeBiopharm, Boerhringer Ingelheim, and OmniSeq [all institutional]): board member (CureMatch, Inc and CureMetrix Inc). AG receives consulting fees from Seattle Genetics and EUSA Pharma.
GF and SS are employees at Foundation Medicine, Inc., a wholly owned subsidiary of Roche Holdings, Inc. and Roche Finance Ltd, and have equity interest in an affiliate of these Roche entities.

The remaining author, TP, declares that he has no competing interests.

\section{Author details}

${ }^{1}$ Center for Personalized Cancer Therapy, University of California San Diego (UCSD), 3855 Health Sciences Drive, La Jolla, CA 92037, USA. ²Division of Blood and Marrow Transplantation, UCSD, 3855 Health Sciences Drive, MC-0960, La Jolla, CA 92093, USA. ${ }^{3}$ Foundation Medicine, Inc, 150 Second Street, Cambridge, MA 02141, USA.

Received: 21 February 2021 Accepted: 29 September 2021 Published online: 12 October 2021

\section{References}

1. Goodman AM, Kato S, Bazhenova L, Patel SP, Frampton GM, Miller V, et al. Tumor mutational burden as an independent predictor of response to immunotherapy in diverse cancers. Mol Cancer Ther. 2017;16(11):2598-608. https://doi.org/10.1158/1535-7163.MCT-17-0386.

2. Wu Y, Xu J, Du C, Wu Y, Xia D, Lv W, et al. The predictive value of tumor mutation burden on efficacy of immune checkpoint inhibitors in cancers: a systematic review and meta-analysis. Front Oncol. 2019;9:1161. https://doi. org/10.3389/fonc.2019.01161.

3. Steuer CE, Ramalingam SS. Tumor mutation burden: leading immunotherapy to the era of precision medicine? JCO. 2018;36(7):631-2. https://doi.org/10.1200/JCO.2017.76.8770.

4. Schrock AB, Ouyang C, Sandhu J, Sokol E, Jin D, Ross JS, et al. Tumor mutational burden is predictive of response to immune checkpoint inhibitors in MSI-high metastatic colorectal cancer. Ann Oncol. 2019;30(7): 1096-103. https://doi.org/10.1093/annonc/mdz134.

5. Fernandez EM, Eng K, Beg S, Beltran H, Faltas BM, Mosquera JM, et al. Cancer-specific thresholds adjust for whole exome sequencing-based tumor mutational burden distribution. JCO Precis Oncol. 2019;3(3):1-12. https://doi. org/10.1200/PO.18.00400

6. Heeke S, Hofman P. Tumor mutational burden assessment as a predictive biomarker for immunotherapy in lung cancer patients: getting ready for prime-time or not? Transl Lung Cancer Res. 2018;7(6):631-8. https://doi. org/10.21037/tlcr.2018.08.04.

7. Garassino M, Rodriguez-Abreu D, Gadgeel S, Esteban E, Felip E, Speranza G, et al. OA04.06 Evaluation of TMB in KEYNOTE-189: pembrolizumab plus chemotherapy vs placebo plus chemotherapy for nonsquamous NSCLC. J Thorac Oncol. 2019;14(10):S216-7. https://doi.org/10.1016/j.jtho.2019.08.427.

8. Langer C, Gadgeel S, Borghaei H, Patnaik A, Powell S, Gentzler R, et al. OA04.05 KEYNOTE-021: TMB and outcomes for carboplatin and pemetrexed with or without pembrolizumab for nonsquamous NSCLC. J Thorac Oncol. 2019;14(10):S216.

9. Fancello L, Gandini S, Pelicci PG, Mazzarella L. Tumor mutational burden quantification from targeted gene panels: major advancements and challenges. J Immunother Cancer. 2019;7(1):183. https://doi.org/10.1186/s4 0425-019-0647-4.

10. Patel SP, Kurzrock R. PD-L1 Expression as a predictive biomarker in cancer immunotherapy. Mol Cancer Ther. 2015;14(4):847-56. https://doi.org/10.11 58/1535-7163.MCT-14-0983.

11. Schlam I, Dyehouse KM, Ward PJ. Application of NCCN guidelines in PD-L1 expression for patients with stage IV non-small cell lung cancer. J Clin Oncol. 2018;36(15_suppl):e15030.

12. Alexandrov LB, Ju YS, Haase K, Van Loo P, Martincorena I, Nik-Zainal S, et al. Mutational signatures associated with tobacco smoking in human cancer. Science. 2016;354(6312):618-22.

13. Boichard A, Pham TV, Yeerna H, Goodman A, Tamayo P, Lippman S, et al. APOBEC-related mutagenesis and neo-peptide hydrophobicity: implications for response to immunotherapy. Oncol Immunol. 2018;24(3):1-12. https:// doi.org/10.1080/2162402X.2018.1550341.

14. Alexandrov LB, Nik-Zainal S, Wedge DC, Aparicio SAJR, Behjati S, Biankin AV, et al. Signatures of mutational processes in human cancer. Nature. 2013; 500(7463):415-21. https://doi.org/10.1038/nature12477.

15. Boichard A, Tsigelny IF, Kurzrock R. High expression of PD-1 ligands is associated with kataegis mutational signature and APOBEC3 alterations. Oncoimmunology. 2017;6(3):e1284719. https://doi.org/10.1080/2162402X.2 017.1284719 . 
16. Gerlinger M, Rowan AJ, Horswell S, Math M, Larkin J, Endesfelder D, et al. Intratumor heterogeneity and branched evolution revealed by multiregion sequencing. N Engl J Med. 2012;366(10):883-92. https://doi.org/10.1056/ NEJMoa1113205.

17. Riaz N, Havel JJ, Makarov V, Desrichard A, Urba WJ, Sims JS, et al. Tumor and microenvironment evolution during immunotherapy with nivolumab. Cell. 2017;171(4):934-949.e16

18. Frampton GM, Fichtenholtz A, Otto GA, Wang K, Downing SR, He J, et al. Development and validation of a clinical cancer genomic profiling test based on massively parallel DNA sequencing. Nat Biotechnol. 2013;31(11): 1023-31. https://doi.org/10.1038/nbt.2696.

19. Johnson DB, Frampton GM, Rioth MJ, Yusko E, Xu Y, Guo X, et al. Targeted next generation sequencing identifies markers of response to PD-1 blockade. Cancer Immunol Res. 2016;4(11):959-67. https://doi.org/10.11 58/2326-6066.CIR-16-0143.

20. Chalmers ZR, Connelly CF, Fabrizio D, Gay L, Ali SM, Ennis R, et al. Analysis of 100,000 human cancer genomes reveals the landscape of tumor mutational burden. Genome Med. 2017;9(1):34

21. Bonta I, Isac JF, Meiri E, Bonta D, Rich P. Correlation between tumor mutation burden and response to immunotherapy. JCO. 2017;35(15_suppl): e14579.

22. Devarakonda S, Rotolo F, Tsao M-S, Lanc I, Brambilla E, Masood A, et al. Tumor mutation burden as a biomarker in resected non-small-cell lung cancer. J Clin Oncol. 2018;14:JCO2018781963.

23. Johnson A, Severson E, Gay L, Vergilio J-A, Elvin J, Suh J, et al. Comprehensive genomic profiling of 282 pediatric low- and high-grade gliomas reveals genomic drivers, tumor mutational burden, and hypermutation signatures. Oncologist. 2017;22(12):1478-90. https://doi.org/1 0.1634/theoncologist.2017-0242.

24. Goodman AM, Sokol ES, Frampton GM, Lippman SM, Kurzrock R Microsatellite-stable tumors with high mutational burden benefit from immunotherapy. Cancer Immunol Res. 2019;7(10):1570-3. https://doi.org/1 0.1158/2326-6066.CIR-19-0149.

25. Chang $L$, Chang M, Chang HM, Chang F. Microsatellite instability: a predictive biomarker for cancer immunotherapy. Appl Immunohistochem Mol Morphol. 2018;26(2):e15-21. https://doi.org/10.1097/PAl. 0000000000000575 .

26. Choi S, Yu Y, Grimmer MR, Wahl M, Chang SM, Costello JF. Temozolomideassociated hypermutation in gliomas. Neuro Oncol. 2018;20(10):1300-9. https://doi.org/10.1093/neuonc/noy016.

\section{Publisher's Note}

Springer Nature remains neutral with regard to jurisdictional claims in published maps and institutional affiliations.

Ready to submit your research? Choose BMC and benefit from:

- fast, convenient online submission

- thorough peer review by experienced researchers in your field

- rapid publication on acceptance

- support for research data, including large and complex data types

- gold Open Access which fosters wider collaboration and increased citations

- maximum visibility for your research: over $100 \mathrm{M}$ website views per year

At $\mathrm{BMC}$, research is always in progress.

Learn more biomedcentral.com/submissions 